\title{
Sobre los orígenes de la ciencia política: la formalización del derecho político en el marco constitucional y administrativo
}

POR CLAUDIO CONTRERAS $(*)$

\begin{abstract}
Sumario: I. Introducción.- II. El surgimiento de la ciencia política en la tratadística del Estado de derecho.- III. La ciencia política del Estado de derecho y el análisis neokantiano de la estatalidad.- IV. El problema de la precedencia del Derecho al Estado.- V. Conclusión: el estatuto gnoseológico del neokantismo en la ciencia política.- VI. Bibliografía.
\end{abstract}

Resumen: en el presente artículo vamos a analizar las primeras formulaciones de la denominada ciencia política a mediados del siglo XIX. La oposición a nivel de la tratadística del Estado de derecho, entre una reducción de la legalidad a la aplicación en la jurisprudencia, como planteaba Laband, y un análisis de los campos factuales de intervención para procedimientos administrativos, como planteaba Von Stein, lleva a un replanteo del problema político subyacente en la discusión jurídica-dogmática. En este marco, la tentativa de formalizar a la política misma reduciendo el marco de conflictos, decisiones y dominios a formas pautadas de proceder, lleva a formular una ciencia política. A diferencia de la Polizeiwissenchaft del siglo XVIII, lo que se plantea aquí no es una regla para la monarquía administrativa, sino la formación de una clase política "de profesión" que permita gestionar los intereses representados por el sistema democrático de partidos.

Palabras claves: ciencia política - positivismo - Kantismo - profesión

On the origins of political science: the formalization of political law in the constitutional and administrative framework

Abstract: in the present article we will make an analysis on the first formulation of the so called "political science" in the middle of the nineteenth century. The opposition that works on the level of the Rule of law theory, between the reduction of the

(*) Abogado, Universidad Nacional de La Plata. Doctor en Ciencias Jurídicas, Universidad Nacional de la Plata. Prof. Titular de Derecho Político, Universidad del Salvador y Universidad Nacional del Noroeste de la Provincia de Buenos Aires (UNNOBA). 
legal problem on the application of the law in the cases of the jurisprudence, that is formulated by Laband, and the analysis of the fields for prosecution by the official administration, defended by Rudolph Von Stein, makes an inevitable reference to the political dimension. The tentative of formalization in politics is posed whit the objective of a general reduction of the conflictive, decisional and arbitrary domain for a transition towards a scientific rules on political procedure. In contrast with the Polizeiwissenchaft of the XVIII century, the political science is not a rule of administrative monarchy, but a technical approach in the formation of the political class, proposed in view of a professionalization of the representative system of parties and negotiation of social interests.

Keywords: political science - positivism - Kantism - profession

\section{Introducción}

En nuestros estudios anteriores analizamos de forma preliminar la formación del Estado de derecho en sus dos aspectos generales: de un lado el derecho constitucional y legislativo, del otro el derecho administrativo. El problema de la articulación de las dos instancias, la dificultad de mantener su funcionamiento y la irreductibilidad del mismo al esquema positivista de la "ley y su aplicación" nos llevaron a plantear el problema del derecho político en el marco de la disputa constitucional. Lo que tenemos que esclarecer en el análisis presente es precisamente el marco epistemológico en el que la tratadística del Estado de derecho va a definirse, no como ciencia de la policía, ni como ciencia del Estado, constitucional o administrativa, sino como ciencia política. Esto nos permitirá ingresar en el segundo aspecto de la cuestión abordada: el pasaje de la legalidad a la administración que determina los problemas del derecho político en su abordaje científico.

La formación de la ciencia política, a mediados del siglo XIX, comprende una serie de modificaciones en el planteo del Rechtstaat: en primer lugar, determina una posición intermedia entre el positivismo legislativo de Laband y el vitalismo evolucionista de Spencer, sin que por ello se identifique con las posturas de Jhering y de Bluntschli. Retoma, más bien, el romanismo historicista de Savigny, y el organicismo funcionalista de Krause (Heuschling, 2002, p. 175). El marco general es aquel del neokantismo a partir del cual se establece una especie de análisis gramatical de las significaciones generales de los conceptos para delimitar luego su referencia a datos empíricos.

Ahora bien, como veremos, este marco neokantiano refiere ante todo al problema de la formación de un sujeto cognoscente y agente: de un lado, este opera en las formaciones históricas divergentes, las cuales realizan de forma parcial los conceptos de Estado y de derecho; pero a la vez, en cuanto son conscientes de 
tales operaciones, pueden modificar sus términos y formar modelos eidéticos de proceder en vistas de perfeccionar la estatalidad. En segundo lugar, se plantea, en cambio, el problema de la evolución propia de tales conceptos en el marco general de la vida: el problema está precisamente en comprender cómo es la persona que porta los derechos y los hace cumplir libremente, sin suponer una coacción externa. Ahora bien, la única forma de hacerlo es precisamente suponiendo que la personalidad jurídica es el Estado mismo: éste es el portador de los derechos y, por ende, quien los hace cumplir libremente.

Los colectivos históricos y su evolución vital divergente pertenecen, entonces, a un marco de estatalidad general en el que tienen lugar y se desarrollan; ahora bien, en cuanto la estatalidad, es esta forma institucional colectiva que actúa y reflexiona de forma racional, la personalidad estatal se da a sí misma instituciones que permiten proceder, y establece un saber de estado cuya forma eminente es la ciencia política. A través de tal fundamentación se determinan al menos dos objetivos: diferenciar las formas gubernamentales y los partidos, de un sector político "de profesión", y reducir a las personas, oficiales y civiles, a sujetos sobre los que se aplica el derecho.

\section{El surgimiento de la ciencia política en la tratadística del Estado de derecho}

Examinemos de forma detenida los principios que rigen a la teoría política con respecto a las formas constitucionales y con respecto a las funciones administrativas que conciernen al Estado. En el derecho político es sabido que el Estado es el objeto principal de las indagaciones y, aunque se trata del Estado considerado desde el punto de vista de su derecho, todo lo que en tal sentido se piense estará supeditado a la definición del Estado. Por otra parte, en la tratadística del Estado de derecho era necesario suponer, cuando se trataba de una exposición sistemática, que los problemas se tenían que examinar siguiendo en lo posible el orden conceptual antes que las clasificaciones históricas vigentes. Y es aquí donde vemos aparecer como una "absoluta necesidad" para toda otra consideración ulterior respecto del Estado, con respecto de cualquier problema en lo tocante a sus relaciones, la determinación adecuada de su "concepto fundamental" (Grundbegriff). De allí que la posición fundadora de la denominada ciencia política en el siglo XIX estableciera un cuestionamiento con respecto a las posiciones políticas "militantes" y diera lugar a la determinación de una definición propia del Estado. Esta posición intermedia entre la práctica empírica de la administración, que propugnaba Lorenz Von Stein, y la analítica dogmática de la jurisprudencia, defendida por Laband, la vino a formular por primera vez Gaspar Bluntschli: 
Cuántas disertaciones se escriben, cuántos libros se publican acerca de la política, o dan como noción preliminar una idea o un concepto del Estado, o los suponen formulados, o todavía prescinden de tal formulación de forma explícita o implícita; cayendo, en este último caso, en el defecto harto general de la superficialidad, de la contradicción y del empirismo. ¿Quién no ve bien claro que el desorden en las ideas y en la conducta de los políticos militantes, aun de los políticos que proceden de buena fe y con sana intención, nace de la ignorancia de la ciencia del Estado? ¿Quién no ve que la inseguridad con que los partidos políticos se conducen, el predominio en la vida real de los Estados históricos de la habilidad personal, el interés inusitado que por cualquier circunstancia adquiere un problema secundario, dependen en gran parte de la falta de ideas y de conceptos claros y definidos del Estado? (Bluntschli, 1880, p. 110)

Para el planteo de Bluntschli, entonces, la participación constante, y por lo mismo superficial, y en cierto modo irreflexiva, que todos tienen como miembros de la colectividad política, no es un requisito para comprender la estatalidad. Para responder a la idea de una conducta política adecuada, en esa esfera amplía en que los miembros del Estado colaboran, bastaría entonces con un conocimiento empírico de las circunstancias que más de cerca nos rodean, bastaría con obrar según lo exija la cualidad de todo acto racional humano. Pero cuando se trata de intervenir en la política, como gobernante, en cambio, sería precisa una ciencia respectiva a una determinada "conducta profesional" donde el concepto definido (no cerrado ni cristalizado) del Estado no puede ser equívoco: en pocas palabras, según Bluntschli, es precisa la ciencia política para dar una determinación unívoca del objeto "Estado", cuya realización sería el fin intencionado del agente. La ciencia política, por ende, se formula, a fines del siglo XIX, como la doctrina del Estado que tienen que conocer los denominados "políticos de profesión".

Por estas razones, entre otras, la determinación del concepto del Estado es el primer problema de la política entendida como una actividad profesional. Formar el concepto del Estado consiste, entonces, en determinar, mediante previo análisis de los elementos y notas que se nos ofrecen a nuestra consideración racional, lo que el mismo es, a diferencia o en distinción con los demás objetos de la realidad. Para conseguir esto es necesario que, teniendo en cuenta la representación que la palabra "Estado" despierta en nosotros fijemos la idea que corresponde a tal representación, para lo cual es preciso que suspendamos toda nuestra atención sobre la palabra misma, examinando cuantos datos y noticias respecto de su propio significado poseemos ya, o mediante la investigación podamos aportar (Blunstchli, 1880, p. 122). Para salir de la formalización positivista de la jurisprudencia y no caer en el empirismo de la administración es preciso un punto de vista gramatical de los conceptos fundamentales. 
De una forma más sutil puede afirmarse que la ciencia política, para formular sus definiciones del Estado, tuvo que enfrentar el dilema positivista que merma entre un criterio de fundamentación del Estado según un modelo ideal abstraído y un criterio genético del Estado según el origen histórico del mismo. Según la doctrina del positivismo (Jellinek, 1900, p. 126), o se rechazaba la posibilidad de la formación del concepto e idea del Estado, si por tal se entiende cosa distinta de una mera generalización de los hechos históricos conocidos, o procediendo en apariencia como lo hacía Aristóteles en su Política (es decir, ateniéndose meramente al análisis genético y al de los fines), el concepto del Estado se formulaba por épocas y al presente se lo limitaba a lo que puede resultar de un estudio comparativo de los Estados contemporáneos. La ciencia política se dedicó a distinguir, entonces, entre los autores historicistas en el análisis del origen del Estado, como Mommsen y aquellos autores, como Hegel, en quienes la influencia idealista a la que aludimos no se manifiesta de un modo claro y terminante, de modo tal que se entiende y se formula el concepto del Estado de suerte que aparece como si esto fuera la investigación de un ideal abstracto, irrealizable en circunstancias de tiempo y de lugar dadas.

Para los enfoques de la ciencia política de fines del siglo XIX y desde allí para todos los análisis del Estado que enfrentaron al dilema positivista, se hizo preciso evitar ambas tendencias, la idealista y la historicista, la fundamentación abstracta y la genética. Bluntschli, tal vez el más notable publicista, cuyas obras políticas estuvieron, desde mediados del siglo XIX, en manos de todos los tratadistas de la doctrina estatal, daba un ejemplo de la doble crítica a estas tendencias, pero también a la tentativa de llevar al extremo, o una consideración puramente formalista, del tipo del "estado constitucional puro" a una consideración puramente sustancialista, del tipo del "estado absolutista de la ciencia de la policía".

Lo que se enfrentaba, entonces, no eran dos teorías del Estado, una positivista y una empirista, una formalizadora y otra tipificante, sino dos formas de gobernar. De un lado la administración monárquico-constitucional, del otro el aparato de justicia fijado en los antecedentes de la jurisprudencia. Para confrontarse al positivismo, Bluntschli distinguía entre la noción o concepto del Estado (Staatsbegrtff) e idea del Estado (Staatsidee):

La noción o concepto del Estado, decía, determina la naturaleza y los caracteres esenciales de los Estados reales; y la idea muestra con el brillo de una perfección ideal el modelo del Estado no realizado todavía, pero que se pretende realizar. Por el estudio de la historia descubrimos el primero; por la especulación filosófica el segundo (Bluntschli, 1880, p. 123). 
Es preciso notar que esta apreciación llevaba a incorporar a la doctrina del Estado elementos histórico-materiales generalizados tal como lo exigían Spencer, Greef y, en general, los sociólogos evolucionistas. Pero la doctrina del Estado de la ciencia política no trataba solo de reducir el concepto del Estado a su formulación sociológico-evolutiva: para los tratadistas consecuentes, entonces, había que tomar importantes reparos frente a la opinión de Bluntschli. En primer lugar, lo que el autor definía como noción o concepto del Estado no se presentaba como otra cosa más que la noción o concepto del Estado histórico, es decir, su Estado contemporáneo, en primer lugar, y los residuos de los antecedentes, en segundo. Esos Estados reales eran los Estados constituidos cuya estructura era extraída y unificada en un modelo por medio de un estudio comparativo de los mismos, que permitía dilucidar la cuestión de la idea o concepto ideal que aquellos suponían.

Pero a la ciencia política no le era suficiente este método comparado, ya que no se llegaría a una conclusión adecuada por una mera generalización de las notas o caracteres en que coincidían constitutivamente todos o la mayoría de los Estados; y dado que, según Bluntschli, se iba en busca de "caracteres esenciales", el problema estaba en determinar cómo es que estos podían hallarse en el curso evolutivo de las historias particulares, sin recurrir a un esquema del tipo hegeliano de la "idea del Estado" en su realización inmanente. Antes bien, para obtener ese resultado, era precisa la determinación previa (explícita o implícita) del concepto fundamental del Estado, como base de un criterio racional que permitiera discernir, en medio de la exterior confusión con que se nos aparece lo histórico, aquello que es esencial (es decir, conforme a la naturaleza ideal no abstracta del Estado) de lo que era meramente transitorio y de lo que entraña un desconocimiento efectivo del Estado mismo. Con esto, la tratadística el Estado de derecho se propuso entonces no una extracción, sino una idealización de modelo que sirviera como plan procedimental.

Para la ciencia política en su formación derivada de la tratadística del Estado de derecho, el dilema entre una concepción positivista formalista y constitucional y una concepción evolutiva sustancial y administrativa, era preciso superar, sin excluirla de forma taxativa, a la fundamental distinción entre el propósito del historiador, cuyo objeto es lo fenomenal positivo, realizado, y el del filósofo, que se propone investigar no lo que el Estado fue en tal o cual momento, sino lo que es su "naturaleza universal". La noción a que aludía Bluntschli no era la del Estado, sino la del Estado histórico en las condiciones en que se vive. Si bien entrañaba un propósito que traspasaba los límites de la historia, no apuntaba su indagación a la investigación de los hechos por lo que ellos valían y como tales significaban, sino que atendía a determinar, según los hechos y mediante una aplicación del procedimiento inductivo, caracteres esenciales. 
En cuanto a lo que Bluntschli decía de la idea del Estado, era preciso radicalizar e invertir su esquema: no tanto abstraer las formas esenciales de los Estados históricos comparándolos según un supuesto modelo previo, sino considerar sus tendencias inmanentes en vistas a la formación, concreta, de un modelo de procedimientos idealizado. Desde luego puede afirmarse que un concepto filosófico del Estado no entraña la investigación de su ideal cuando por ideal se entiende el modelo del Estado no realizado todavía, pero que se pretende realizar. El dilema estaba precisamente en separar la política militante de partidos fraccionados, para distinguir una política protocolar en la que se opere según "profesionalismo". Así, cuando por ciertos políticos se combate la manía teórica idealista, en virtud de la cual parece que en la política real todo es imperfección y limitación, habiendo necesidad de buscar en un supuesto ideal la posibilidad de un porvenir perfecto absoluto. La ciencia política buscaba este modelo ideal frente a las tendencias parciales neutralizadas:

No se trata entonces de referir la misión del que forma un concepto del Estado en la búsqueda de perfecciones irrealizables, ni menos atender sólo a una posibilidad de realización, remota en el tiempo. Así, para investigar al Estado (y la determinación del concepto es el primer paso necesario), no se puede mirar al porvenir remoto, ni al pasado, ni al presente, porque todo esto implica limitación, posición determinada definida, o lo que es igual, realización de ideal mediante adaptación del mismo a circunstancias dadas. Suponer que en ellas se le contiene todo, es suponer que el ideal se agote, o lo que es igual, desconocerlo en su negación (Von Jhering, 1947, p. 140).

Para la ciencia política la determinación del concepto del Estado posible en un porvenir que habría de ser alguna vez efectivo, imponía a aquel las mismas limitaciones que si se tratara de fijar el estado propio del momento actual. El porvenir, solo por serlo, no se presentaba como mejor que el presente o el pasado y el ideal no indicaba una realización remota, por necesidad, sino un modelo operativo de procedimientos cuya tendencia era encontrada en los Estados históricos analizados. Para la ciencia política, el ideal del Estado, como todo ideal, en cuanto significaba la esencia de las cosas que se realiza en los hechos, no era patrimonio de nadie, ni era definido para tiempo alguno determinado. Notemos, sin embargo, que no se trata, como en el hegelianismo, de encontrar ya operante ese ideal en el curso histórico, con mayor o menor perfección, sino de proponerlo como modelo operativo. En otros términos, la ciencia política no se proponía encontrar una idea de Estado en el curso histórico, presente, pasado o futuro, sino construir un modelo aplicable a casos determinados.

De este modo, la ciencia política podía retomar los problemas del evolucionismo y del positivismo y rescatarlos, a pesar de esas limitaciones con que la 
determinación del Estado aparece en Bluntschli y Holtzendorff, pero también las de Laband y Stahl, en cuanto había en tales autores no pocos datos aprovechables para fijar el concepto del Estado y en cuanto, para ello, se requiriera un análisis racional de los fenómenos políticos. Para formar este análisis racional de los fenómenos políticos en vistas a un modelo operativo de los procedimientos de Estado, la ciencia política procedía desde el lugar del sujeto de conocimiento, buscando, mediante la reflexión, un criterio, no cerrado y definitivo, sino amplio y abierto siempre, para determinar de un modo adecuado lo que el Estado, de la política, es o debe ser. Esto no quería decir que se pretendiera sacar en cada situación subjetiva de conocimiento, como en una generación espontánea, el Estado como una invención de la tratadística, tal como lo pretendían los modelos ideales de la filosofía; antes bien, como el Estado es cosa que efectivamente se verifica en el tiempo, en cuanto su condición se pone, las fuentes de conocimiento y para el conocimiento del Estado se comprendían como dadas en la relación a la propia conciencia y reflexión con el mundo exterior.

En este sentido, el peligro más temible que era preciso evitar para poder proceder a la investigación científica y elevar la política al estatuto de una "ciencia" estaba en lo que se venía a denominar como el "dogmatismo personal", en cierto modo subjetivo, que llevaba, en política especialmente, a dar como verdades inconcusas opiniones más o menos admisibles y a sentar como ideas salvadoras, como panaceas que curan todos los males sociales, fórmulas cerradas, que cada cual sacara de su propia "sustancia espiritual". En este sentido, el problema de la filosofía estaba en plantear un estado "cerrado" que, desde Fichte a Hegel, se presentaría como la forma última de la "idea" en su desarrollo:

El Estado de derecho de la ciencia política es un proyecto "abierto": si en la ciencia en general sería un absurdo, como advertía Lange, hacer afirmaciones definitivas, concretas y terminantes, en la política aparece ese absurdo más palmario, desde el punto de vista teórico, y sus consecuencias prácticas son en extremo lamentables. Precisamente esa definición cerrada de las ideas en la ciencia es lo contrario de lo que se busca en el concepto y de cuanto se podía investigar en toda filosofía. Las condiciones de la realidad misma y de la propia manera de ser espiritual hacen que toda definición, como toda afirmación concreta, sean siempre posiciones limitadas, fenomenales, manifestaciones que nunca agotan el ideal (Von Jhering, 1947, p. 213).

Pero el concepto mismo del "ideal" es lo que estaba mutando: en principio, la formulación es equivalente a la hegeliana, según la cual nuestra propia naturaleza racional tiene como característica una cualidad sublime, que la capacita para elevarse por encima de cuanto significa limitación y agotamiento definitivo, comenzando por salir de la esfera mezquina de la individualidad para considerar lo 
universal y superior, en que comulgan al fin los hombres todos. La noción idealista parece a su vez rectificada por la frase de Griner, según la cual "la racionalidad es el poder de ser y vivir más allá de lo limitado y de la hora presente en lo ilimitado y en todas las horas". Pero en verdad la ciencia política formulaba un modelo operativo eidético, acorde a los términos del neokantismo de Ritter y no un ideal absoluto propio del idealismo de Fichte, Schelling o Hegel.

Partiendo de tal analítica inmediata de las significaciones conscientes para llegar a las formas gramaticales inconscientes e indicar de qué manera se entiende la determinación del concepto del Estado, tal era la metodología de la ciencia política en sus años de formación: acudiendo a los datos que en la propia conciencia puedan ser contrastados y analizados, se procuraba distinguir aquel objeto de la indagación en la realidad en que cada individuo vive y se mueve. La intención estaba, entonces, en determinar aquello en que consiste esencialmente el Estado, según resulta del análisis reflexivo de lo que ya se sabía del mismo: tomar los datos de experiencia, no de modo puro, sino como formas elaboradas de conceptos de la conciencia, y de allí comprender a los procedimientos en vistas a una meta racional eidética.

\section{La ciencia política del Estado de derecho y el análisis neokantiano de la estatalidad}

La ciencia política, entonces, establece un análisis que refiere a los caracteres lingüísticos con respecto al modo en que generan significaciones en la conciencia del sujeto cognoscente:

La palabra Estado tiene el significado único con que muchas otras palabras se nos presentan, especialmente las palabras técnicas y las que, como advierte Stuart Mill, fueron inventadas por modo expreso después de la idea o la cosa que se les hace expresar. Antes bien, Estado despierta en nosotros en primer término la idea de una significación amplísima, que sólo desde un punto de vista puede tener algo que ver con la política o con lo político. "Estado" vale tanto como manera de ser o de estar las cosas, como posición determinada: refiere al mero hecho de ser en cuanto algo está siendo. Por tanto, significa lo contrario al mudar que significa el cambio, en cierto modo, el movimiento. Pero si nos fijamos, las ideas del estado y del mudar que, aunque expresan cosas diferentes, entrañan una íntima e indisoluble relación. En efecto, el mudar supone el cambio de estados, y el estado es forma del mudar que se mantiene en el ser. La ordenada, o más bien orgánica compenetración de esta idea, afirma la vida, la cual no indica sino la permanencia de algo persistente y característico del ser a través de los 
diversos estados sucesivos en que el ser mismo se manifiesta (o muda) (Jellinek, 1900, p. 264).

Esta idea lingüístico-significativa de estado, tan amplia, tan comprensiva, según los analistas de la ciencia política, arrojaba una nueva luz sobre la idea del Estado del derecho político. En realidad, este Estado particular implicaría, como el estado en su amplio sentido, la idea de situación, de modo tal que, si cada uno conscientemente se fijara en lo que el común sentir quiere indicar cuando habla del Estado refiriéndose a la política, se notaría que alude implícita o explícitamente a la situación, a la manera de ser o de estar la colectividad humana, considerada bajo una cierta forma. De este modo, del análisis lingüístico se pasaba, entonces, a una investigación detenida del significado histórico de la palabra Estado, en vistas a determinar si esta lleva a una similar conclusión.

Una segunda base para el conocimiento científico, entonces, no es la idea general del significado de la palabra, sino el modo en que esta se refiere a una instancia institucional históricamente determinada: si "estado" supone una situación, un modo de mantenerse en el ser a través de la mutación, el "estado político", tiene que remitir la situación a una colectividad determinada caracterizada por ser "política". En efecto, el análisis se va a servir, no de las formas eidéticas del neokantismo, sino de la romanística del siglo XIX, desde Savigny a Niebhur. Un ejemplo claro de una colectividad que refiere la situación política es la historia romana que analiza Niebhur. Para este, la idea del Estado residía en la cosa pública, lo perteneciente a la sociedad como cuerpo, que era lo que más atraía la atención, lo que con más unidad y con mayor persistencia se cuidaba, hasta el punto de absorber casi por completo toda actividad libre, de modo que el Estado venía a significar la situación de la res publica, y por antonomasia indicaba el estado del todo social. Y, según decía el historiador romanista, aunque en un principio en la historia romana, "Estado" se dijese más bien de la condición personal del ciudadano, porque el conjunto de instituciones que determinaban semejante condición era la Ciudad, la extensión que al fin alcanzaría el Imperio y, aun la universalización de aquella condición o estado de ciudadanía, llevarían a una modificación general de las significaciones. El término ciudad, que indicó siempre círculo social limitado territorialmente, se hizo impropio para designar el sistema de condiciones en que una vida política social tan extensa se realizaba y el Estado vino a ser el medio en el cual la condición se hacía efectiva. Estado, entonces, según Niebhur (y tal como lo retoma Von Jhering), poniéndose en analogía con la estatalidad de las monarquías constitucionalizadas del siglo XIX, sería para Roma imperial algo como el orden de la vida pública de todo un pueblo o masa de hombres socialmente organizada (Von Jhering, 1947, p. 316).

Para los análisis de la ciencia política, la palabra Estado aplicada a fenómenos reales de naturaleza particular, se especializa en su significado propio por virtud 
de la importancia que adquiere el Estado o situación de la cosa pública. En el fondo, el Estado de la política no expresa una idea distinta de aquella que se expresa con su significado más amplio, según la cual, aquello que en la historia viene a enunciarse con el Estado político es anterior a la admisión de semejante acepción gramatical del término "Estado". En otros términos, la evolución histórica, según la cual en cada período respectivo se extiende la situación de estatalidad política a las diversas formas colectivas e institucionales, es el índice que permite aplicar el significado gramatical general a una multiplicidad de casos diversos, ya que, según afirmaban los tratadistas, "conocemos el Estado porque lo vivimos" (Von Jhering, 1947, p. 254).

Pero la ciencia política no restringe a tal aplicación de la gramática el significado histórico final: para penetrar el sentido histórico del concepto del Estado, antes de limitarse a lo expuesto de forma final, es preciso exponerlo en su modo evolutivo. Si Hegel decía que de las formas primitivas de la colectividad se daba una evolución de la idea del Estado que permitía ir de la familia a la sociedad civil y de ésta a la estatalidad jurídica y moral, para las ciencias políticas, en cambio, es el hecho de que ciertas sociedades históricas hayan absorbido a las colectividades diversas en una forma institucional generalizada lo que da pie a la estatalidad. Es por la determinación de lo público como forma social autónoma y por la absorción de ésta sobre las instancias "privadas" que la "familia" surge como familia, como unidad íntegra, como entidad autónoma, que la religión deviene una institución con aspiraciones universales, la cual, por la intensidad y originalidad de su acción, se diferencia idealmente del Estado. Surgen también las asociaciones municipales como otros tantos Estados, aparecen los diversos pueblos, se forman más adelante los gremios, las corporaciones de todo género y, ya en los tiempos cercanos a nosotros, se constituyen las diversas sociedades libres para fines determinados de la vida humana y lentamente brota la individualidad, afirmando su propia y esencial sustantividad e independencia. En este sentido, las diversas colectividades sociales son discernibles como tales y articulables entre sí, en cuanto se generan formas de estatalidad que las incluyan, como subordinadas - sean públicas o privadasen la forma general de una institución superior.

En este punto Von Jhering se separa de los análisis historicistas de Niebuhr, ya que, si es de tal contingencia histórica que - en determinadas sociedades- lleva a la formación de la estatalidad, cabe cuestionar en qué queda el Estado en medio de toda esa desintegración social a la que, casi de pasada, se alude, y si una institución tan importante como el Estado puede desmoronarse de una vez por sí. Si tal cosa sucediera, no sería precisa la determinación de su concepto, pues ese fenómeno nos indicaría que estábamos en presencia de una de esas formaciones sociales transitorias. Sin embargo, para la ciencia política esto no es así: el hecho factual de que, una vez desmoronadas las formas de estatalidad antiguas, se 
den períodos de desintegración en colectividades separadas, no anula el hecho de que la estatalidad tenga una autonomía y que pueda resurgir (Von Jhering, 1947, p. 315).

El lazo político, entonces, no es solo una situación institucional de colectividades desarrolladas que se puede constatar históricamente: es también una forma autoconsciente de colectividad que supone, en vistas a su desarrollo, la formación inmanente de una ciencia. Para la ciencia política, el hecho de que en las sociedades primitivas no se nos presenten organizaciones políticas especiales, bajo formas propias, con aquella complicación de funciones específicas y aquella desintegración de elementos con que los Estados de las sociedades civilizadas se presentan, no obsta para poder afirmar la existencia de un Estado en ellas. El Estado, como cuanto es, se realiza en medio de un conjunto de condiciones que lo determinan y modifican. De allí que el problema factual de que la situación histórica lleve siempre a formas imperfectas y parciales de estatalidad. Es en ello, precisamente, que encuentra su justificación la existencia de una ciencia política. En otros términos, para la ciencia política, si bien es verdad que la evolución histórica antes indicada echaba luz para penetrar en el fondo de lo que el Estado significaba como situación colectiva específica, también lo era el hecho de que la marcha seguida por aquella misma evolución, por una parte, llevaría a la realización imperfecta y limitadísima del Estado, y por otra conduciría a su negación parcial en ocasiones.

Para una analítica de pretensión científica, la variedad de principios, que espontánea o reflexivamente se producen en los diversos pueblos, tales como el del Self-government en Inglaterra, los derechos del hombre en Francia, el Rechtsstat en Alemania y las Nacionalidades en España - entre otros- suponen ya una idealización de una forma de gobierno. En otros términos, la ciencia política no solo justifica la necesidad de una reflexión autoconsciente de los procedimientos que pueden hacer que la estatalidad subsista e integre a las colectividades diversas que la preceden, sino que tiene lugar allí donde la misma estatalidad se presenta en un marco limitado como Estado de derecho y no como un ejercicio desmedido del gobierno y de la autoridad personal. La ciencia política tiene que ajustar la situación política de la estatalidad a los medios de la constitucionalidad y la administración del Estado de derecho.

\section{El problema de la precedencia del Derecho al Estado}

Así, de lo expuesto por la ciencia política se desprende una consecuencia fundamental con respecto a la índole del Estado de derecho, según el cual este no consiste en la limitación del poder del Estado por el derecho que se le interpone, sino más bien el despliegue del derecho por los medios del Estado. En otros 
términos, la dependencia en que el Estado se encuentra respecto del derecho y la necesidad de determinar, ante todo, lo que es el derecho, para poder saber en qué consiste al fin el Estado. De allí que la ciencia política se sirva del modelo romano como forma ideal, pero que también pase a encontrar el derecho en cualquier formación social, por más primitiva que fuera. En tal sentido es que va a aplicarse al Estado lo que Von Stein decía del derecho:

Siempre se ha mostrado la idea del derecho como un resorte fundamental, como un principio determinante de la vida social humana y de todas sus instituciones, como una fuerza viva. Ninguna otra necesidad se ha dejado con mayor energía ni más pronto en todas épocas que la de construir un orden jurídico cualquiera, por más que la idea de este orden, en los grados inferiores de la civilización, no haya sido ni podido ser comprendida durante largo tiempo en toda su naturaleza, sino en tal o cual de sus aspectos tan sólo, y al principio únicamente en el más exterior, flotando en su espíritu como un presentimiento más o menos velado, e incapaz de expresarse en otra forma que en la de la costumbre, tan irregular e insegura (Von Stein, 1859, p. 193).

El análisis gramatical de los conceptos fundamentales de la ciencia jurídica, entonces, lleva a que, de un lado, se supone una situación política factual y del otro, la exigencia de un derecho, de modo tal que se vuelva innecesario insistir ya más en señalar la estrecha e íntima relación que existe entre las ideas del Estado y del derecho. Teniendo en cuenta lo que antes se afirmaba sobre el Estado, como institución para el derecho, se hacía preciso explicar detenidamente lo que al fin con el lema del Estado de derecho se venía a indicar, para lo cual era preciso tener presente siempre la acepción amplia del concepto de Estado. Según tal análisis, el Estado político no indica sólo la manera de ser o de estar - estática- de la mutación, del sistema de condiciones dadas que constituyen el orden político, sino también el movimiento, la actividad que en la misma cosa pública se manifiesta: es el elemento permanente, que, persistiendo como tal, se realiza o pone en serie de Estados:

El Estado viene a ser, según esto, como el derecho (en su contenido esencial) en cuanto se cumple en la vida. Aplicando aquí términos que tienen un significado estricto en otras ciencias, podría decirse que el Estado viene a ser, en cierto respecto, el órgano (el instrumento vivo en sí mismo) de la función jurídica o del derecho, y la aplicación va a parecer la más adecuada y propia para la ciencia política. En efecto; ese contenido esencial, que no está quieto e inmóvil, sino que entraña un principio de actividad, requiere, como todos los de su índole, un medio y forma para obrar, para hacer efectiva en toda circunstancia y momento su actividad. Como responde a una necesidad esencial de 
la vida del conjunto social y la actividad que supone tiende a la satisfacción de aquélla, de ahí que lo llamemos función, y que llamemos órgano al Estado. Si bien es de advertir que la palabra órgano puede no ser adecuada para expresar todos los momentos históricos de su vida, sobre todo si se le considera como órgano específico constituido. (Jellinek, 1900, p. 289).

Nos encontramos, entonces, con la formación de un concepto organicista del Estado que no se reduce ni a su análisis biológico ni al corporativismo jurídico: bajo el lema del Estado de derecho, entonces, la ciencia política expone el modo en que la situación política de la estatalidad viene a operar la función jurídica y ponerla en acto. En otros términos, se pasa a considerar el modelo evolucionista del organismo y sus funciones, junto con aquel historicista de la personalidad estatal que daba la romanística: la amplia significación que organismo tiene después Krause y Spencer especialmente, por lo que sociedad puede considerarse como un organismo, viene a ser aunada a la forma jurídica de la personalidad abstracta que proponían los romanistas desde Savigny hasta Niebhur (Jellinek, 1900, p. 300).

\section{Conclusión: el estatuto gnoseológico del neokantismo en la ciencia política}

En resumen, puede afirmarse como resultado, que el Estado se ofrece a la ciencia política desde fines del siglo XIX como el orden político (no público) que aparece como institución o conjunto de instituciones permanentes fundadas históricamente. Sin embargo, el saber de Estado, su ciencia, tiene que generar modelos ideales que expliquen el lazo que une a los hombres en determinadas circunstancias para ordenar su vida colectiva de un modo adecuado, según un sistema, más o menos completo, de condiciones libres y recíprocas. En breves términos: el Estado viene a ser institución para el derecho, pero la institución solo se despliega a través de la formación de un saber del Estado.

Para la ciencia política, entonces, ni la imperfección del saber, en sociedades primitivas o precivilizadas, ni la imperfección del poder, en aparatos autoritarios y puramente gubernamentales, a través de la confusión social de los pueblos considerados "incultos" o "despóticos", impiden que se vea siempre el Estado (o como el respectivo pueblo lo llame) regulando la vida social. Del mismo modo, en medio de la complicada vida de las sociedades modernas, el Estado no desempeña otra función y más preciso aún es la formación de una ciencia autoconsciente que regule al proceder y de un derecho explícito que límite al poder. De allí, por último, que la ciencia política al asumir para sí a la tratadística del Estado de derecho acabe por cuestionarse que, aun si al decir que el Estado es institución para el derecho, no se entienda nada de lo tocante a la naturaleza, dirección y extensión de 
su fin, el lazo político al que responde, no puede ser sí mismo sino el lazo jurídico por excelencia, de modo tal que vivir políticamente no puede ser sino vivir en el derecho y que el hombre (ser de razón) no podría vivir si no es como ser político, según ya decía Aristóteles, más comprendiéndose ahora esto como ser capaz de derecho.

Es preciso notar aquí un punto de inflexión en la tratadística histórica: las ciencias de la policía del siglo XVIII, las ciencias del Estado de la primera mitad del siglo XIX, derivan en la formación de una ciencia política en cuanto tratan de absorber las oposiciones del formalismo positivista y del historicismo evolucionista. De allí que sus análisis no se opongan a las conclusiones parciales a que los historiadores del "hombre primitivo" y los sociólogos del siglo XIX llegaban, ya que, analizando los elementos sociales de las tribus más lejanas en civilización e instituciones de la civilización e instituciones modernas, a fines del siglo XIX se señalará algo que ya no será la idea rousseauniana del "buen salvaje". Se referirá, en cambio, el hecho de que allí donde se encuentre la necesidad política, esencial en el hombre, se encontrará también como más esencial y definida sea esta, y cuanto más desenvuelta esté su cualidad de racional y libre, más se verá aunada a una forma jurídica. La ciencia política que inicia en el siglo XIX, y tal como la definía Malberg, supone que de la persona como sujeto práctico y cognoscente que pertenece a la esfera de la vida, el Estado es precisamente el aspecto jurídico de la misma, es su personalidad jurídica:

En cierto sentido puede afirmarse que hay una institución para el derecho; el derecho en rigor no se produce, como se produce el trigo, o el hierro; el derecho se vive, y si se dice que el Estado lo realiza, no puede suponerse que el Estado elabora derecho que luego la sociedad consume, sino que la vida de la persona se ordena y desenvuelve jurídicamente, siendo en tal función, la persona que por sí misma sabe ordenarse y desenvolverse, verdadero Estado" (Carré de Malberg, 1895, p. 142).

En otros términos, la ciencia política tiene que concebir al Estado como la persona jurídica que se desenvuelve libremente en su actuar y racionalmente en su pensar: este nivel jurídico es precisamente un nivel de la "vida" (Heuschling, 2002, p. 214). En otros niveles, en cambio, se puede considerar a la persona psicológica, cultural, biológica, etc. y desde allí a la persona individual, pero, en el marco de la estatalidad, la persona que precede a los individuos es el Estado. Sin embargo, lo paradójico de esta concepción reside en que, aun manteniendo la noción jurídica de personalidad, se la pretende incluir en la esfera de la vida y a la vez excluirla de la misma en sus aspectos específicos, es decir, no jurídicos. Como veremos, aquí es precisamente que surge el problema que la ciencia política no podrá resolver en términos meramente conceptuales. Se trata de que la situación factual del Estado, 
su "estado o factualidad política", no coincide con la personalidad jurídica que representa, del mismo modo que el derecho como campo de sujeción coactivo no coincide con la vida sobre la que se aplica.

\section{Bibliografía}

Bluntschli, G. (1880). Deutsche Staatslehre und die heutige Staatenwelt. Berlin: Nördlingen.

Carré de Malberg, R. (1895). Contribution à la théorie générale de l'État. Paris: CNRS Éditions.

Heuschling, L. (2002). État de droit, Rechtsstaat, rule of law. Paris, Dalloz.

Jellinek, G. (1900). Allgemeine Staatslehre. Berlin, Hölder.

Von Stein, L. (1852). System der Staatswissenschaft. Basilea: Statistik.

Von Jhering, R. (1947). Tres estudios jurídicos. Buenos Aires: Atalaya.

Fecha de recepción: 01-04-2019 Fecha de aceptación: 12-09-2019 